Résumés des conférences et travaux

Art et archéologie de la Chine pré-impériale

\title{
La Chine et les routes de la soie
}

Meicun Lin

\section{(2) OpenEdition}

Journals

Édition électronique

URL : https://journals.openedition.org/ashp/1057

DOI : $10.4000 /$ ashp. 1057

ISSN : 1969-6310

Éditeur

Publications de l'École Pratique des Hautes Études

Édition imprimée

Date de publication : 2 février 2011

Pagination : 327-330

ISSN : 0766-0677

\section{Référence électronique}

Meicun Lin, "La Chine et les routes de la soie ", Annuaire de l'École pratique des hautes études (EPHE), Section des sciences historiques et philologiques [En ligne], 141 | 2011, mis en ligne le 25 février 2011 consulté le 06 juillet 2021. URL : http://journals.openedition.org/ashp/1057 ; DOI : https://doi.org/ 10.4000/ashp. 1057 


\title{
LA CHINE ET LES ROUTES DE LA SOIE
}

\author{
Conferences de M. LiN Meicun, \\ université de Pékin, \\ directeur d'études invité conjointement \\ par MM. Étienne de LA VAIssière et Alain Thote
}

\section{Une carte de la route de la Soie du XVI siècle découverte récemment au Japon}

En 2002, un musée japonais a vendu une peinture de ses réserves à peu près inconnue des spécialistes. Ce rouleau horizontal, large de $59 \mathrm{~cm}$ et long de $30,12 \mathrm{~m}$, est peint sur soie. Endommagé partiellement à l'endroit où se trouvent habituellement un titre et des colophons, il avait été identifié par les conservateurs du musée comme une peinture de paysage des Qing (1644-1911). Pourtant, une étiquette était collée au dos du rouleau, indiquant ceci : «Carte-paysage de la Mongolie ». Il s'agit bien d'une carte, mais du XVI ${ }^{\mathrm{e}}$ siècle et représentant les territoires anciennement traversés par la route de la Soie. C'est aux différents problèmes posés par cette carte, à l'histoire de sa transmission, à son contenu et sa forme matérielle qu'a été consacrée la première conférence. Le territoire couvert s'étend de Jiayuguan dans l'actuel Gansu à la mer Rouge, et deux cent onze noms y sont portés en chinois, pour la plupart des transcriptions de noms mongols, ouïgours, persans, arabes, ou latins. Il a été d'abord démontré que sa fabrication se situe entre les années 1524 et 1539. La cartographie chinoise utilise traditionnellement plusieurs modes de représentation, dont celui du paysage. Cette carte a été peinte dans le style de l'école de Wu (Suzhou et sa région), en bleu et vert. Par comparaison avec d'autres cartes reproduites dans des livres xylographiés, le territoire qu'elle couvrait dans sa forme initiale devait s'étendre jusqu'à Istanbul. Elle apporte cependant des informations qui ne figurent pas dans les versions gravées, par exemple sur l'observatoire de Samarkand créé en 1420 et celui de Dengfeng au Henan. Ce dernier, construit en 1276, sur l'ordre de Kubilaï (1214-1294), fut restauré sous Qianlong au XVIII ${ }^{\mathrm{e}}$ siècle. Ici, il est restitué dans son aspect d'origine. Malgré l'intérêt présenté par ces sites, on observe dans la carte la présence de motifs standard repris de différentes peintures du XVI ${ }^{\mathrm{e}}$ siècle, par exemple d'un maître de l'école de Wu comme Qiu Ying (1498-1552). Ces rapprochements confirment la date de la Carte-paysage de Mongolie ainsi que l'identification de Suzhou comme lieu de production. Elle offre en outre une synthèse des connaissances géographiques qu'avaient réunies les Ming au-delà des frontières de leur empire.

\section{Nouvelles données archéologiques concernant la diffusion du bouddhisme en Asie centrale}

Le premier témoignage de l'arrivée du bouddhisme au Turkestan est un sutra en gāndhāri, le Dharmapada ou « Stances de la Loi » (Fajujing), écrit sur écorce de bouleau. 
Découvert à Hetian (Khotan), il date au plus tard du $\mathrm{II}^{\mathrm{e}}$ siècle de notre ère. Or plusieurs découvertes récentes sont venues s'ajouter au corpus des traces anciennes d'influence bouddhiste et indienne dans le bassin du Tarim. Cette conférence a eu pour but de les présenter. On sait qu'au Gāndhāra, les plus anciens objets portant la marque de l'art bouddhique sont des tablettes à fard en vogue durant l'ère scytho-parthe. Or, récemment, deux tablettes de ce type ont été découvertes à Hetian et à Kashgar, montrant que dès avant le $\mathrm{II}^{\mathrm{e}}$ siècle l'art bouddhique avait atteint le Turkestan. Une découverte plus importante a été faite en 2003 par des pillards dans la partie est du bassin du Tarim, près de la capitale du royaume de Kroraina, en chinois Loulan (site LE) : il s'agit d'une tombe à décor peint datée du III $^{\mathrm{e}}$ siècle. La structure de la tombe est chinoise et le cercueil est décoré de motifs chinois. La tombe se situe en cela dans le prolongement de la diffusion des coutumes funéraires chinoises attestées dans le corridor du Hexi à l'époque Han, et on en possède d'autres exemples dans le bassin du Tarim. Mais les peintures des murs et du pilier de la tombe sont dans le style du Gāndhāra, et représentent des scènes de banquet et des processions bouddhiques avec des dédicaces en kharoshti. Les banqueteurs, portant des caftans et de longues barbes, sont certainement des habitants de Loulan. Dans l'iconographie de la tombe, les représentations de la Roue de la Loi bouddhique sont particulièrement mises en avant, figurant à de nombreuses reprises sur le pilier central. Une représentation du Bouddha en robe rouge entouré d'orants est un autre signe du caractère bouddhique de l'iconographie. On souligne les parallèles avec l'art contemporain du monastère de Fayaz Tépé en Ouzbékistan. Les liens des habitants du Sud du bassin du Tarim avec l'empire Kouchan sont discutés d'après les textes historiques chinois et les manuscrits fragmentaires découverts au début du $\mathrm{XX}^{\mathrm{e}}$ siècle : sur les murs de la tombe on trouve en effet une inscription kharoshti, peut-être une signature, qu'il faut mettre en relation avec l'adoption dans tout le royaume de Loulan de ce système d'écriture indien, sans doute sous l'influence de l'empire Kouchan. Dernier élément rapprochant cette iconographie du Gāndhāra : le thème du banquet et du vin, également représenté sur l'une des tablettes à fard, fort bien représenté dans le monde indo-iranien. Une robe fragmentaire en soie a été également découverte, sur laquelle figurait Dionysos.

\section{III. À la recherche de Gengis Khan}

Au début du XIII ${ }^{\mathrm{e}}$ siècle, Gengis Khan (1162 ?-1227) conquit l'ensemble du continent eurasiatique. À l'occasion du $800^{\mathrm{e}}$ anniversaire de la création de son empire, le Musée d'histoire nationale de Chine a organisé en 2006 une mission de prospection en République populaire de Mongolie. La conférence a porté sur les résultats de cette mission, en particulier sur les vestiges des sites occupés par Gengis Khan. Plusieurs découvertes ont été faites au $\mathrm{XX}^{\mathrm{e}}$ siècle. À Uglugchiin Kherem, un rempart, dit « de Gengis Khan », situé sur le versant sud-est de la montagne Daichin, daterait en fait de l'époque des Liao (907-1125) d'après la présence de tessons chinois. Il protège un cimetière, et non une ville. D'autres sites, comme celui de Zu Kherem, montrent également une filiation entre les premières constructions des Mongols aux XI ${ }^{\mathrm{e}}$ et XII ${ }^{\mathrm{e}}$ siècles et celles des Liao, qu'elles imitent. Plusieurs stèles érigées en Mongolie commémorent de grands événements de son histoire. Leurs textes ont été présentés et les informa- 
tions qu'ils contiennent, confrontées à ce que les sources chinoises nous rapportent. La seconde partie de la conférence a été consacrée à la présentation de trois des résidences de Gengis Khan, en commençant par celle de Halaotu à Salichuan, sa résidence d'été. D'après la cartographie ancienne, l'emplacement de ce palais correspondait à un lieu-dit, « le lac aux deux sources » (Gun Nuar, qui signifie « lac profond»). De fait, deux sources jaillissent à Salichuan, l'une d'eau douce, l'autre d'eau salée. De ce palais ne subsiste qu'un mur d'enceinte, et à peu de distance à l'ouest de la vieille ville, trois petits bâtiments. La beauté du site naturel où il fut construit avait inspiré à la fin du XII ${ }^{\mathrm{e}}$ siècle l'aménagement du palais de l'empereur Zhangzong des Jin à Pékin. Ici encore il existe des liens étroits entre les constructions mongoles et celles des dynasties occupant le Nord de la Chine sous les Song (960-1268). Un autre palais, dit de printemps, Khuduu Aral, fut la plus grande des quatre résidences de Gengis Khan. Il a été découvert à Avraga en 1961 et fouillé en 2001 par une équipe de Mongols et de Japonais. Les fouilles ont révélé quatre niveaux d'occupation, dont l'un comprend les vestiges de la terrasse des étendards, que l'on a comparée à une représentation figurant sur une peinture chinoise anonyme du musée de Boston (Le retour en Chine de Wenji). La conférence s'est achevée sur une discussion concernant le mausolée des empereurs de la dynastie Yuan à Qiniangu. Ce toponyme donné par les sources chinoises est probablement la transcription du nom Gurelgu, un massif situé dans la région du Chentej Nuruu.

\section{Découverte de Tamao, l'île où les Portugais accostèrent pour la première fois en Chine en 1514}

La localisation de l'île de Tamao où les Portugais accostèrent pour la première fois en Chine est l'objet d'un débat animé parmi les spécialistes du fait que le nom de Tamao n'est que la transcription du mot qui désignait un « grand port » dans la langue de l'époque, et non un lieu précis. Les nouvelles découvertes archéologiques ont démontré que les premiers témoignages du trafic de porcelaines opéré par les Portugais se trouvent sur l'île de Shangchuan au Guangdong. Les Portugais avaient coutume à chaque fois qu'ils exploraient une nouvelle route de navigation de marquer leur point d'arrivée par une pierre dressée appelée padrâo ou « stèle maritime ». Plusieurs sont toujours debout, par exemple au Kenya. Les sources écrites rapportent que les Portugais débarquèrent pour la première fois en Chine sur l'île de Tamao et qu'ils y érigèrent un padrâo. Une carte dont l'original remonte au $\mathrm{XVI}^{\mathrm{e}}$ siècle montre d'ailleurs l'île de Tamao dans le delta de la rivière des Perles portant un padrâo. De plus, on sait depuis longtemps qu'outre les textes portugais, une source chinoise semble bien mentionner la colonne historiée qu'était un padrâo. En 2006, une mission archéologique de repérage s'est rendue sur l'île de Shangchuan. La mission a constaté que le site était parsemé de tessons de porcelaine «bleu et blanc» datant de la première moitié du $\mathrm{XVI}^{\mathrm{e}}$ siècle, mais surtout elle a découvert un padrâo, conservé à hauteur d'homme quoique très érodé. Il s'agit certainement de celui élevé en 1514 par le Portugais Jorge Alvarez, qui fut d'ailleurs enterré à son pied. L'île de Shangchuan semble donc bien être le lieu où les Portugais abordèrent pour la première fois en Chine, et à partir duquel ils tentèrent d'approcher la cour chinoise afin d'obtenir l'autorisation de 
commercer. La conférence a d'une part permis de présenter les résultats de la mission de 2006, en particulier les céramiques et le padrâo, et d'autre part de dresser l'inventaire des sources écrites, portugaises et chinoises, traitant de l'arrivée des Portugais en ce lieu et plus largement du contexte politique et militaire (personnel portugais de l'expédition, mais aussi par exemple les luttes locales contre la piraterie japonaise, et le devenir de l'implantation). 